Publ. Mat. 47 (2003), 195-209

\title{
VIRTUALLY REPELLING FIXED POINTS
}

\author{
Xavier Buff
}

\begin{abstract}
In this article, we study the notion of virtually repelling fixed point. We first give a definition and an interpretation of it. We then prove that most proper holomorphic mappings $f: U \rightarrow V$ with $U$ contained in $V$ have at least one virtually repelling fixed point.
\end{abstract}

\section{Preliminaries}

Let $f:(\mathbb{C}, \alpha) \rightarrow(\mathbb{C}, \alpha)$ be a holomorphic germ fixing $\alpha$.

Definition 1. The multiplicity $m$ of $\alpha$ as a fixed point of $f$ is the residue

$$
m=\text { residue }\left(\frac{1-f^{\prime}(z)}{z-f(z)} d z, \alpha\right) .
$$

In other words, it is the multiplicity of $\alpha$ as a root of $z-f(z)$. When $m=1, \alpha$ is a simple fixed point and when $m>1, \alpha$ is a multiple fixed point.

The multiplier of $f$ at $\alpha$ is the derivative $\lambda=f^{\prime}(\alpha)$. When $|\lambda|>1$, $\alpha$ is repelling, when $|\lambda|<1, \alpha$ is attracting and when $|\lambda|=1, \alpha$ is indifferent.

If $\alpha$ is repelling or multiple, it is weakly repelling.

Remark. Observe that $\alpha$ is multiple if and only if $\lambda=1$.

One easily proves that analytic conjugacy preserves the multiplier at a fixed point and that topological conjugacy preserves the property of being repelling, attracting or indifferent. Topological conjugacy preserves the multiplier at an indifferent fixed point (see $[\mathbf{N}]$ or $[\mathbf{P M}]$ ). It also preserves the multiplicity of a fixed point and two germs having multiple fixed points with the same multiplicity are always topologically conjugate (see $[\mathbf{C}]$ ). 
Definition 2. The residue fixed point index $\iota(f, \alpha)$ of $f$ at a fixed point $\alpha$ is the residue

$$
\iota(f, \alpha)=\operatorname{residue}\left(\frac{1}{z-f(z)} d z, \alpha\right) .
$$

If the multiplicity is $m$ and $\Re(\iota(f, \alpha))<m / 2, \alpha$ is virtually repelling, if $\Re(\iota(f, \alpha))>m / 2, \alpha$ is virtually attracting and if $\Re(\iota(f, \alpha))=m / 2, \alpha$ is virtually indifferent.

It is known that the residue fixed point index is invariant under analytic conjugacy (see [M, Lemma 12.3]) but not under topological conjugacy (as mentioned above, two germs having a multiple fixed point with the same multiplicity are always topologically conjugate). Since the notions introduced above are invariant under analytic conjugacies, it makes sense to talk about the multiplier or the residue fixed point index of a holomorphic germ $f:(U, \alpha) \rightarrow(U, \alpha)$ where $U$ is an arbitrary Riemann surface and $\alpha \in U$ is an arbitrary point (for example the point $\infty$ in $\mathbb{P}^{1}$ ).

In Section 2, we show that a virtually repelling fixed point with multiplicity $m$ may be thought as the superposition of $m$ fixed points which are repelling on average. More precisely, we prove the following theorem.

Theorem 1. A germ $f:(\mathbb{C}, \alpha) \rightarrow(\mathbb{C}, \alpha)$ has a virtually repelling fixed point at $\alpha$ if and only if any sufficiently small perturbation $f_{\varepsilon}$ of $f$ has at least one virtually repelling fixed point close to $\alpha$. Besides, if $\alpha$ has multiplicity $m$ and is not virtually repelling, there exist arbitrarily small perturbations $f_{\varepsilon}$ having $m$ attracting fixed points close to $\alpha$.

In $[\mathbf{J}]$, Jellouli proves that when $P(z)=e^{2 i \pi p / q} z+z^{2}$, then

$$
\frac{q+1}{2}-2^{q-1} \leq \Re\left(\iota\left(P^{\circ q}, 0\right)\right) \leq \frac{q+1}{2} .
$$

The upper bound says that 0 is a virtually repelling fixed point of $P^{\circ q}$. The proof relies on the fact that the multiplicity of 0 as a fixed point of $P^{\circ q}$ is $q+1$ and that there exist small perturbations $P_{\varepsilon}$ of $P$ such that $P_{\varepsilon}^{\circ q}$ has only repelling fixed points. In $[\mathbf{S h} 3],[\mathbf{B}]$ and $[\mathbf{B E}]$, refinements of this result are given.

In Sections 3 and 4, we prove that most ramified coverings $f: U \rightarrow V$ with $U \subset V$ have at least one virtually repelling fixed point.

Definition 3. Let $U \subset V$ be Riemann surfaces and $f: U \rightarrow V$ be a holomorphic map. We say that $f$ is repelling on average if and only if $f$ has finitely many fixed points $\alpha_{k} \in U$ with multiplicities $m_{k}$ and

$$
\Re\left(\sum \iota\left(f, \alpha_{k}\right)\right)<\frac{1}{2} \sum m_{k} .
$$


When a holomorphic map $f: U \rightarrow V$ is repelling on average, the barycenter of the quantities $\iota\left(f, \alpha_{k}\right) / m_{k}$ weighted with multiplicities $m_{k}$ has real part less than $1 / 2$. Therefore, one of those quantities must have real part less than $1 / 2$. Hence, $f$ must have at least one virtually repelling fixed point.

Let us first consider the case where $U=V=\mathbb{P}^{1}$, i.e., the case of rational maps. It is well-known (see [M, Section 10]) that for any rational map $f: \mathbb{P}^{1} \rightarrow \mathbb{P}^{1}$, we have the equality

$$
\sum_{\left\{\alpha \in \mathbb{P}^{1} \mid \alpha=f(\alpha)\right\}} \iota(f, \alpha)=1 .
$$

This is known as the Fatou's Index Formula, or the Holomorphic Fixed Point Formula. When the degree of $f$ is $d \geq 2$, it has $d+1$ fixed points counted with multiplicity. Therefore $f$ is repelling on average and has at least one virtually repelling fixed point. In Sections 3 and 4, we generalize this result.

In Section 3, we introduce the notion of rational-like mappings (see $[\mathbf{R} 1]$ and $[\mathbf{R 2}])$. Those are ramified coverings $f: U \rightarrow V$ where $U$ and $V$ are planar Riemann surfaces with finite Euler characteristic and $U$ is relatively compact in $V$. We prove an analog of Douady-Hubbard's Straightening Theorem (see $[\mathbf{D H}]$ ). We then prove the following result.

Theorem 2. If $f: U \rightarrow V$ is a rational-like mapping, then it has at least one virtually repelling fixed point. Besides, if $V$ is simply connected, $f$ is repelling on average.

Question. Is a rational-like mapping always repelling on average?

In Section 4, we still consider the case where $f: U \rightarrow V$ is a ramified covering with $U \subset V$. We allow $U$ not to be compactly contained in $V$, but we restrict to the case where $V$ is simply connected. In that case, $f: U \rightarrow V$ is conformally conjugate to a ramified covering $g: U^{\prime} \rightarrow \mathbb{D}$ (via an isomorphism $\varphi: V \rightarrow \mathbb{D}$ ). Therefore, we may restrict our study to ramified coverings $f: U \rightarrow \mathbb{D}$ with $U$ contained in $\mathbb{D}$.

Theorem 3. Let $f: U \rightarrow \mathbb{D}$ be a proper holomorphic map of degree $d \geq 2$ with $U$ contained in $\mathbb{D}$. If $|f(z)-z|$ is bounded away from zero as $z \in U$ tends to $\partial U$, then, $f$ has d fixed point in $U$, counting multiplicities, and $f$ is repelling on average. In particular, it has at least one virtually repelling fixed point.

Finally, in Section 5, we give some applications. In particular, we show that if $f$ is a rational map with a (super)attracting fixed point $\alpha$ whose immediate basin $\Omega_{\alpha}$ is not simply connected, then $\Omega_{\alpha}$ separates 
two virtually repelling fixed points of $f$. We also show that when a rational map $f$ has a fixed Herman ring $A$, each connected component of $\mathbb{P}^{1} \backslash A$ contains a virtually repelling fixed point.

\section{Perturbations of virtually repelling fixed points}

In this section we try and understand the notion of virtually repelling fixed points. First, observe that this notion is finer than the one of weakly repelling fixed point. Indeed, when $\alpha$ is not a multiple fixed point, then the residue fixed point index $\iota(f, \alpha)$ and the multiplier $\lambda$ are related by

$$
\iota(f, \alpha)=\frac{1}{1-\lambda} .
$$

Consequently, $|\lambda|>1$ if and only if $\Re(\iota(f, \alpha))<1 / 2$ and a simple fixed point is virtually repelling if and only if it is repelling.

When $\alpha$ is a multiple fixed point of multiplicity $m$ and $\Re(\iota(f, \alpha))<$ $m / 2$, we think of $\alpha$ has being the superposition of $m$ fixed point which are repelling on average. This is essentially the content of Theorem 1 which says that a germ $f:(\mathbb{C}, \alpha) \rightarrow(\mathbb{C}, \alpha)$ has a virtually repelling fixed point at $\alpha$ if and only if any sufficiently small perturbation $f_{\varepsilon}$ of $f$ has at least one virtually repelling fixed point close to $\alpha$. In particular, if all the fixed point of $f_{\varepsilon}$ are simple, at least one of them is repelling.

Proof of Theorem 1: First, the result is clear if $\alpha$ is a simple fixed point. Now, assume $\alpha$ is a multiple fixed point and let $m$ be its multiplicity. The multiplicity and the residue fixed point index of $f$ at $\alpha$ can be defined via the integrals of $\left(1-f^{\prime}(z)\right) /(z-f(z))$ and $1 /(z-f(z))$ along a small loop $\gamma$ turning once around $\alpha$. Those integrals depend continuously on $f$. It follows that for any sufficiently small perturbation $f_{\varepsilon}$ of $f$, the sum of the multiplicities $m_{k}$ at the fixed point $\alpha_{k}$ of $f_{\varepsilon}$ contained in the region delimited by $\gamma$ is equal to $m$ and the sum of residue fixed point indices of $f_{\varepsilon}$ at the points $\alpha_{k}$ is close to the residue fixed point index of $f$ at $\alpha$. In particular, for any sufficiently small perturbation $f_{\varepsilon}$, we have

$$
\Re\left(\sum \iota\left(f_{\varepsilon}, \alpha_{k}\right)\right)<\frac{1}{2} \sum m_{k} .
$$

This precisely means that the barycenter of the quantities $\iota\left(f_{\varepsilon}, \alpha_{k}\right) / m_{k}$ weighted with multiplicities $m_{k}$ has real part less than $1 / 2$. In particular one of those quantities has real part less than $1 / 2$ and the corresponding fixed point is virtually repelling.

Conversely, assume $\alpha$ is not virtually repelling. We will show that there exist arbitrarily small perturbations $f_{\varepsilon}$ having $m$ attracting fixed points close to $\alpha$. We will obtain the perturbation in two steps. 
Step 1: If $\alpha$ is virtually indifferent, we first make a perturbation that turns it into a virtually attracting fixed point. For example, consider the perturbation $f_{\varepsilon}:(\mathbb{C}, \alpha) \rightarrow(\mathbb{C}, \alpha)$ defined by

$$
\frac{1}{z-f_{\varepsilon}(z)}=\frac{1}{z-f(z)}+\frac{\varepsilon}{z-\alpha} .
$$

The map $f_{\varepsilon}$ has a fixed point of multiplicity $m$ at $\alpha$ and the residue fixed point index is

$$
\iota\left(f_{\varepsilon}, \alpha\right)=\iota(f, \alpha)+\varepsilon .
$$

When $\Re(\varepsilon)>0$, the fixed point becomes virtually attracting.

Step 2: Without loss of generality, we may now assume that $\alpha$ is virtually attracting, i.e., $\Re(\iota(f, \alpha))>m / 2$.

In order to get a hand on the residue fixed point index, we will use the following fact, which is known but not absolutely obvious (see for example the appendix in $[\mathbf{B E}]$ ): one may perform an analytic change of coordinates so that the Taylor expansion of $f$ at $\alpha$ becomes

$$
f(z)=\alpha+(z-\alpha)+(z-\alpha)^{m}+\iota(f, \alpha)(z-\alpha)^{2 m-1}+\mathcal{O}\left(|z-\alpha|^{2 m}\right) .
$$

Let us first study the case of the polynomial $g: \mathbb{C} \rightarrow \mathbb{C}$ defined by

$$
g(z)=\alpha+(z-\alpha)+(z-\alpha)^{m}+\iota(f, \alpha)(z-\alpha)^{2 m-1} .
$$

We define $\lambda_{\varepsilon}$ by

$$
\frac{1}{1-\lambda_{\varepsilon}}=\frac{i}{\varepsilon}+\frac{\iota(f, \alpha)}{m}
$$

and set $g_{\varepsilon}=\alpha+\lambda_{\varepsilon}(g-\alpha)$. The fixed points of the polynomials $g_{\varepsilon}$ are the solutions of the equation

$$
1+(z-\alpha)^{m-1}+\iota(f, \alpha)(z-\alpha)^{2(m-1)}=\frac{1}{\lambda_{\varepsilon}} .
$$

For small values of $\varepsilon$, the polynomial $g_{\varepsilon}$ has $m$ attracting fixed points close to $\alpha$ : one at $\alpha$ with multiplier $\lambda_{\varepsilon}$ and $\alpha_{1}, \ldots, \alpha_{m-1}$ which, by symmetry, all have the same multiplier. Thus, the residue fixed point index $\iota\left(g_{\varepsilon}, \alpha_{k}\right)$ does not depend on $k=1, \ldots, m-1$. Now, we have

$$
\iota\left(g_{\varepsilon}, \alpha\right)=\frac{i}{\varepsilon}+\frac{\iota(f, \alpha)}{m}
$$

and

$$
\iota\left(g_{\varepsilon}, \alpha\right)+\sum_{k=1}^{m-1} \iota\left(g_{\varepsilon}, \alpha_{k}\right) \underset{\varepsilon \rightarrow 0}{\longrightarrow} \iota(f, \alpha) .
$$


Therefore, for all $k=1, \ldots, m-1$, we have

$$
\iota\left(g_{\varepsilon}, \alpha_{k}\right)=\frac{-i}{(m-1) \varepsilon}+\frac{\iota(f, 0)}{m}+o(1) .
$$

In particular, for small values of $\varepsilon$, the residue fixed point indices have real part close to $\Re(\iota(f, \alpha)) / m>1 / 2$ and the fixed points are attracting.

We will now define the perturbation of $f$. Let us work in a local coordinate where $f(z)=g(z)+\mathcal{O}\left(|z-\alpha|^{2 m-1}\right)$. We define the perturbation $f_{\varepsilon}$ by

$$
\frac{1}{z-f_{\varepsilon}(z)}=\frac{1}{z-g_{\varepsilon}(z)}+\frac{1}{z-f(z)}-\frac{1}{z-g(z)} .
$$

The fixed point of $f_{\varepsilon}$ are the poles of $1 /\left(z-f_{\varepsilon}(z)\right)$. Since

$$
\frac{1}{z-f(z)}=\frac{1}{z-g(z)}+\mathcal{O}(1)
$$

the fixed points of $f_{\varepsilon}$ which are close to $\alpha$ and their residue fixed point indices coincide with the fixed point of $g_{\varepsilon}$ which are close to $\alpha$ and their residue fixed point indices. In particular, for small values of $\varepsilon, f_{\varepsilon}$ has $m$ attracting fixed points close to $\alpha$.

Remark. The proof given above also shows that when $f:(\mathbb{C}, \alpha) \rightarrow(\mathbb{C}, \alpha)$ has a virtually repelling fixed point at $\alpha$ with multiplicity $m$, there exist arbitrarily small perturbations $f_{\varepsilon}$ having $m$ repelling fixed points close to $\alpha$.

\section{Rational-like mappings}

Definition 4. A rational-like mapping is a proper holomorphic mapping $f: U \rightarrow V$ of degree $d \geq 2$, where $U$ and $V$ are connected open subsets of $\mathbb{P}^{1}$ with finite Euler characteristic and $U$ is relatively compact in $V$.

A rational-like mapping comes with a filled-in Julia set $K_{f}$ (the set of non-escaping points), and a Julia set $J_{f}$ (the boundary of $K_{f}$ ). The Julia set $J_{f}$ may equivalently be defined as the closure of the set of repelling periodic points. The following result shows that rational-like mappings behave like rational maps. This result is probably not new and may already appear somewhere in the literature.

Theorem 4 (Straightening Theorem). For any rational-like map $f: U \rightarrow$ $V$, there exist a rational map $F: \mathbb{P}^{1} \rightarrow \mathbb{P}^{1}$, neighborhoods $U^{\prime}$ and $V^{\prime}$ of the Julia set $J_{F}$ and a quasi-conformal homeomorphism $\varphi: V \rightarrow V^{\prime}$ which conjugates $f: U \rightarrow V$ to $F: U^{\prime} \rightarrow V^{\prime}$. 
Remark. The $\bar{\partial}$ derivative of $\varphi$ may be chosen to vanish everywhere on $K_{f}$.

Proof: We will only sketch the main lines of the proof of Theorem 4 . The proof mimics the one by Douady and Hubbard of the straightening theorem for polynomial-like mappings (see $[\mathbf{D H}]$ ).

By restricting $V$ if necessary, we may assume that $U$ and $V$ have smooth boundaries. We denote by $\left(B_{i}\right)_{i \in \mathcal{I}}$ the connected components of $\mathbb{P}^{1} \backslash V$ and by $\left(D_{j}\right)_{j \in \mathcal{J}}$ the connected components of $\mathbb{P}^{1} \backslash U$. The sets $\mathcal{I}$ and $\mathcal{J}$ are finite by assumption, and the sets $B_{i}$ and $D_{j}$ are Jordan domains. Besides, each $B_{i}$ is contained in a unique $D_{j}$, and the inclusion induces a map $\iota: \mathcal{I} \rightarrow \mathcal{J}$. The rational-like map $f$ induces a mapping $f_{*}: \mathcal{J} \rightarrow \mathcal{I}$ so that $f\left(\partial D_{j}\right)=\partial B_{f_{*}(j)}$.

We may now define an extension of $f$ to the Riemann sphere. This extension will be quasi-regular and will satisfy Shishikura's principle (see [Sh1, Lemma 1]). Therefore, the proof will be completed.

If $\left(j_{0}, j_{1}, \ldots, j_{n}\right)$ is a periodic cycle of the map $\iota \circ f_{*}$, we define $A_{j_{k}}$ to be the annulus $\bar{D}_{j_{k}} \backslash B_{f_{*}\left(j_{k-1}\right)}$ and we let $d_{j_{k}}$ be the degree of $f \mid$ $\partial D_{j_{k}}$. We choose a real number $\left.r \in\right] 0,1\left[\right.$, and we define $A_{j_{k}}^{\prime}$ to be the annulus $\left\{z \in \mathbb{C}\left|r^{d_{k-1}} \leq\right| z \mid \leq r\right\}$. We choose quasi-conformal homeomorphisms $\varphi_{j_{k}}: A_{j_{k}} \rightarrow A_{j_{k}}^{\prime}$ which satisfy $\varphi_{j_{k}} \circ f=\left(\varphi_{j_{k-1}}\right)^{d_{j_{k-1}}}$ on $\partial D_{j_{k-1}}$. Then, we use $\varphi_{j_{k}}$ to glue the dynamics of $z \mapsto z^{d_{j_{k}}}$ in each disk $D_{j_{k}}$. This defines the extension of $f$ in every disk $D_{j}$ such that $j$ is a periodic point of the map $\iota \circ f_{*}$. For the remaining disks $D_{j}$, we choose any quasi-regular extension.

Since any rational map $F$ has at least one weakly repelling fixed point, and since this point is contained in $J_{F}$, it follows that any rational-like map has at least one weakly repelling fixed point. However, since quasiconformal conjugacies do not necessarily preserve the property of being virtually repelling, one has to work a little to prove Theorem 2 .

Proof of Theorem 2: Let us first prove that any rational-like mapping has at least one virtually repelling fixed point. If this were not the case, we could find a rational-like mapping $f: U \rightarrow V$ having only virtually attracting and virtually indifferent fixed points. The idea is to find a perturbation $f_{\varepsilon}: U^{\prime} \rightarrow V^{\prime}$ which is still rational-like but the fixed points of which are all attracting, which clearly gives a contradiction.

Denote by $\alpha_{1}, \ldots, \alpha_{n} \in U$ the fixed points of $f$. In a neighborhood of each fixed point $\alpha_{k}$, Theorem 1 provides a local perturbation $f_{\varepsilon, k}$ which only has attracting fixed points. The only difficulty consists in gluing the perturbations $f_{\varepsilon, k}$ into a global one. For any meromorphic 
function $h: U \rightarrow \mathbb{P}^{1}$, we can write a decomposition $h=\mathcal{P}(h)+\mathcal{R}(h)$, where $\mathcal{P}(h)$ is the polar part of $h$ (i.e., sum of negative powers of $\left(z-p_{j}\right)$ at the poles $p_{j}$ ), and $\mathcal{R}(h) \in \mathcal{O}(1)$ is the regular part of $h$. Then, in a neighborhood of each fixed point $\alpha_{k}$, the polar part $\mathcal{P}\left(1 /\left(z-f_{\varepsilon, k}\right)\right)$ converges to the polar part $\mathcal{P}(1 /(z-f))$ at $\alpha_{k}$ as $\varepsilon$ tends to 0 . Therefore, we can define a global perturbation $f_{\varepsilon}: U \rightarrow \mathbb{P}^{1}$ by

$$
\frac{1}{z-f_{\varepsilon}}=\sum_{k=1}^{n} \mathcal{P}\left(\frac{1}{z-f_{\varepsilon, k}}\right)+\mathcal{R}\left(\frac{1}{z-f(z)}\right) .
$$

The perturbation $f_{\varepsilon}$ is defined on the whole set $U$ but a priori, it is not rational-like. However, since $f_{\varepsilon}$ converge to $f$ on every compact subset of $U$ as $\varepsilon$ tends to 0 , we can find a restriction $V^{\prime}$ of $V$ so that $U \subset V^{\prime} \subset V$ and $f_{\varepsilon}: U^{\prime}=f_{\varepsilon}^{-1}\left(V^{\prime}\right) \rightarrow V^{\prime}$ is rational-like. The fixed points of $f_{\varepsilon}: U^{\prime} \rightarrow V^{\prime}$ and their residue fixed point indices coincide with the fixed points in $U^{\prime}$ of the maps $f_{\varepsilon, k}$ and their residue fixed point indices. By construction, all those points are attracting which gives the required contradiction.

Let us now prove that any rational-like mapping $f: U \rightarrow V$, with $V$ simply connected, is repelling on average. In that case, $f: U \rightarrow V$ is conjugate, via an isomorphism $\varphi: V \rightarrow \mathbb{D}$, to a rational-like mapping $g: U^{\prime} \rightarrow \mathbb{D}$. The number of fixed points of $f$ and $g$ are the same, and the residue fixed point indices coincide. Thus, without loss of generality, we may assume that $V=\mathbb{D}$.

Then, we may choose $r<1$ sufficiently close to 1 so that $U$ is contained in the disk $\mathbb{D}_{r}$ centered at 0 with radius $r$. We set $U_{r}=f^{-1}\left(\mathbb{D}_{r}\right)$ so that $f: U_{r} \rightarrow \mathbb{D}_{r}$ is a ramified covering of degree $d$. For $r$ is sufficiently close to 1 , the boundary of $U_{r}$ is a union of $\mathbb{R}$-analytic Jordan curves (canonically oriented by $U_{r}$ ) and $f$ is holomorphic in a neighborhood of $\overline{U_{r}}$.

Observe that the fixed point of $f$ are contained in $U_{r}$. We must show that $f: U_{r} \rightarrow \mathbb{D}_{r}$ has $d$ fixed points counting multiplicities. For any $z \in \partial U_{r}$, we have $|z|<|f(z)|$. Thus, by Rouché's Theorem, $f$ and Id $-f$ have the same number of zeros in $U_{r}$, counting multiplicities. Since $f: U_{r} \rightarrow \mathbb{D}_{r}$ is a ramified covering of degree $d$, 0 has $d$ pre-images counted with multiplicities. Therefore, there are $d$ fixed points.

We must now show that $f$ is repelling on average. The sum of residue fixed point indices is given by the integral

$$
\frac{1}{2 i \pi} \int_{\partial U_{r}} \frac{d z}{z-f(z)} .
$$


We must prove that the real part of this integral is less than $d / 2$. We can decompose it as follows:

$$
\begin{aligned}
\frac{1}{2 i \pi} \int_{\partial U_{r}} \frac{d z}{z-f(z)} & =\frac{1}{2 i \pi} \int_{\partial U_{r}} \frac{1-f^{\prime}(z)}{z-f(z)} d z+\frac{1}{2 i \pi} \int_{\partial U_{r}} \frac{f^{\prime}(z)}{z-f(z)} d z \\
& =\frac{1}{2 i \pi} \int_{\partial U_{r}} \frac{f^{\prime}(z)}{f(z)} d z+\frac{1}{2 i \pi} \int_{\partial U_{r}} \frac{f(z)}{z-f(z)} \frac{f^{\prime}(z)}{f(z)} d z \\
& =\frac{1}{2 i \pi} \int_{\partial U_{r}} \frac{z}{z-f(z)} \frac{f^{\prime}(z)}{f(z)} d z .
\end{aligned}
$$

Now, $f: \partial U_{r} \rightarrow \partial \mathbb{D}_{r}$ is orientation preserving, and thus, $\frac{1}{2 i \pi} \frac{f^{\prime}(z)}{f(z)} d z$ is real and positive. Besides, when $z \in \partial U_{r}$, we have $|f(z) / z|<1$ and thus

$$
\Re\left(\frac{z}{z-f(z)}\right)<\frac{1}{2} .
$$

Therefore,

$$
\begin{aligned}
\Re\left(\int_{\partial U_{r}} \frac{z}{z-f(z)} \cdot \frac{1}{2 i \pi} \frac{f^{\prime}(z)}{f(z)} d z\right) & =\int_{\partial U_{r}} \Re\left(\frac{z}{z-f(z)}\right) \cdot \frac{1}{2 i \pi} \frac{f^{\prime}(z)}{f(z)} d z \\
& <\frac{1}{2}\left(\frac{1}{2 i \pi} \int_{\partial U_{r}} \frac{f^{\prime}(z)}{f(z)} d z\right)=\frac{d}{2} .
\end{aligned}
$$

This concludes the proof of Theorem 2 .

In fact, still under the assumption that $V$ is simply connected, one can better control the sum of residue fixed point indices. Let $A \subset V$ be the connected component of $V \backslash \bar{U}$ which is not compactly contained in $V$. Observe that $A$ is an annulus. There exists a unique real $\rho \in$ ] $0,1[$ such that $A$ is conformally equivalent to the annulus $\mathbb{D} \backslash[0, \rho]$. The sum of residue fixed points indices of $f$ is contained in the disk of diameter $[d \rho /(\rho-1), d \rho /(\rho+1)]$. The proof is very similar to the argument given above. Without loss of generality, we may assume that $V=\mathbb{D}$ and $0 \in U$. Since the annulus $A$ is conformally equivalent to $\mathbb{D} \backslash[0, \rho]$, it is known (see $[\mathbf{A}$, Section 4]) that for any $z \in U$, we have $|z|<\rho$. Thus, when $z \in \partial U_{r}, z /(z-f(z))$ belongs to the disk of diameter $[\rho /(\rho-1), \rho /(\rho+1)]$. Since $\frac{1}{2 i \pi} \frac{f^{\prime}(z)}{f(z)} d z$ defines on $\partial U_{r}$ a 
positive measure of total mass $d$, the quantity

$$
\frac{1}{d} \int_{\partial U_{r}} \frac{z}{z-f(z)} \cdot \frac{1}{2 i \pi} \frac{f^{\prime}(z)}{f(z)} d z
$$

is a barycenter of the quantities $z /(z-f(z))$. Thus, it still belongs to the disk of diameter $[\rho /(\rho-1), \rho /(\rho+1)]$ and the result is proved.

\section{Proof of Theorem 3}

We now consider the case of a ramified covering $f: U \rightarrow \mathbb{D}$ of degree $d \geq 2$ with $U \subset \mathbb{D}$. We assume that $|f(z)-z|$ remains bounded away from 0 as $z \in U$ tends to $\partial U$. We will prove that $f$ is repelling on average. We will generalize the proof given in the settings of rational-like mappings.

Since $|z-f(z)|$ is bounded away from 0 as $z$ tends to $\partial U, f$ has finitely many fixed point in $U$. Therefore, for any $r$ sufficiently close to 1 , all the fixed points are contained in the disk $\mathbb{D}_{r}$ and we may count them or compute the sum of residue fixed point indices via the integrals

$$
\frac{1}{2 i \pi} \int_{\partial U_{r}} \frac{1-f^{\prime}(z)}{z-f(z)} d z \text { and } \frac{1}{2 i \pi} \int_{\partial U_{r}} \frac{1}{z-f(z)} d z,
$$

where $U_{r}=f^{-1}\left(\mathbb{D}_{r}\right)$. The problem is that we may fail to have $|z|<$ $|f(z)|$ on $\partial U_{r}$ and thus, we must be careful.

In order to prove that $f$ has $d$ fixed points in $U$, we have to redo the proof of Rouché's Theorem. For $r$ sufficiently close to 1 and any $t \in[0,1]$, the function $z \mapsto t z-f(z)$ does not vanish on $\partial U_{r}$. Otherwise, the assumption that $|z-f(z)|$ remains bounded away from 0 as $z$ tends to $\partial U$ would be violated. It follows that for any $r$ sufficiently close to 1 , the integral

$$
I(t)=\frac{1}{2 i \pi} \int_{\partial U_{r}} \frac{t-f^{\prime}(z)}{t z-f(z)} d z
$$

is well defined. It depends continuously on $t$ and takes values in $\mathbb{Z}$. Thus, it is constant. For $t=0$ it gives the number of pre-images of 0 , i.e., the degree of $f$, and for $t=1$ it gives the number of fixed points of $f$. Hence, $f$ has $d$ fixed points in $U$, counting multiplicities.

Next, we have seen that

$$
\sum_{\{\alpha \in U \mid \alpha=f(\alpha)\}} \iota(f, \alpha)=\frac{1}{2 i \pi} \int_{\partial U_{r}} \frac{z}{z-f(z)} \frac{f^{\prime}(z)}{f(z)} d z .
$$

In order to go further with this equality, we would like to do the change of variable $w=f(z)$. Since $f: \partial U_{r} \rightarrow \partial \mathbb{D}_{r}$ is $d$ to 1 , we have to be careful. The ramified covering $f: U \rightarrow \mathbb{D}$ has finitely many critical values in $\mathbb{D}$. 
Thus, if $r_{0}$ is sufficiently close to $1, f: U \backslash \overline{U_{r_{0}}} \rightarrow \mathbb{D} \backslash \overline{\mathbb{D}_{r_{0}}}$ is a non-ramified covering. We define $A$ to be the slit annulus

$$
A=\mathbb{D} \backslash\left\{\overline{\mathbb{D}_{r_{0}}} \cup\left[r_{0}, 1\right]\right\},
$$

and we let $g_{1}, \ldots, g_{d}: A \rightarrow U$ be the $d$ inverse branches of $f$. Then, we can make the change of variables $z=g_{k}\left(r e^{i \theta}\right)$, summing over the $d$ inverse branches:

$$
\sum_{\{\alpha \in U \mid \alpha=f(\alpha)\}} \iota(f, \alpha)=\sum_{k=1}^{d} \frac{1}{2 \pi} \int_{0}^{2 \pi} \frac{g_{k}\left(r e^{i \theta}\right)}{g_{k}\left(r e^{i \theta}\right)-r e^{i \theta}} d \theta .
$$

Since $g_{k}: A \rightarrow U$ is a bounded holomorphic function, a theorem of Fatou asserts that the radial limit of $g_{k}\left(r e^{i \theta}\right)$ exists for almost every $\left.\theta \in\right] 0,2 \pi[$ and that this radial limit is contained in $\partial U$. Thus, by Lebesgue dominated convergence theorem, we may write

$$
\sum_{\{\alpha \in U \mid \alpha=f(\alpha)\}} \iota(f, \alpha)=\sum_{k=1}^{d} \frac{1}{2 \pi} \int_{0}^{2 \pi} \frac{g_{k}\left(e^{i \theta}\right)}{g_{k}\left(e^{i \theta}\right)-e^{i \theta}} d \theta,
$$

where $g_{k}: \partial \mathbb{D} \rightarrow \partial U$ is defined almost everywhere by the radial limit of $g_{k}$. We have $\left\|g_{k}\left(e^{i \theta}\right)\right\|_{\infty} \leq 1$ and as a consequence

$$
\Re\left(\sum_{\{\alpha \in U \mid \alpha=f(\alpha)\}} \iota(f, \alpha)\right) \leq \frac{d}{2} .
$$

In order to get the strict inequality, we must show that we cannot have $\left|g_{k}\left(e^{i \theta}\right)\right|=1$ for every $k=1, \ldots, d$ and almost every $\left.\theta \in\right] 0,2 \pi[$. If this were the case, the boundary of $U$ would be entirely contained in $\partial \mathbb{D}$ and we would have $U=\mathbb{D}$. But then, the ramified covering $f: U \rightarrow \mathbb{D}$ would be a Blaschke product and $|z-f(z)|$ could not remain bounded away from 0 as $z$ tends to $\partial U$.

\section{Applications}

Let us first give some applications of Theorem 2. The following result is due to Przytycki $[\mathbf{P r}]$ if one replaces virtually repelling by weakly repelling. His proof is completely different. We do not think his arguments can yield the existence of virtually repelling fixed points.

Proposition 1. Assume $f: \mathbb{P}^{1} \rightarrow \mathbb{P}^{1}$ is a rational map having a (super)attracting fixed point $\alpha$. If the immediate basin $\Omega_{\alpha}$ of $\alpha$ is not simply connected, it separates two virtually repelling fixed points of $f$. 
Proof: Choose simply connected neighborhoods $U_{0}$ and $U_{1}$ of $\alpha$ such that $U_{0}$ is compactly contained in $U_{1}$ and $f: U_{1} \rightarrow U_{0}$ is a proper mapping (for example, choose a small disk in linearizing or Böttcher coordinates). Then, define $U_{n}$ - by induction - to be the connected component of $f^{-1}\left(U_{n-1}\right)$ which contains $U_{n-1}$. The immediate basin $\Omega_{\alpha}$ is the union of the sets $U_{n}$. Since $\Omega_{\alpha}$ is not simply connected, there exists an integer $n_{0}>0$ such that $U_{n_{0}}$ is simply connected but not $U_{n_{0}+1}$. Define $V=\mathbb{P}^{1} \backslash U_{n_{0}}$. Then, $V$ is connected and simply connected, but $f^{-1}(V)$ has several connected components $V_{1}^{\prime}, \ldots, V_{k}^{\prime}$ compactly contained in $V$. It follows from Proposition 2 that each connected component $V_{j}^{\prime}$ must contain at least one virtually repelling fixed point of $f$.

Let us illustrate this proposition with two examples. The first example is due to Przytycki $[\mathbf{P r}]$ : when $f$ is the Newton's method of a polynomial $P, \infty$ is the unique fixed point which is not attracting. It follows that the immediate basins of attractions of the roots of $P$ are necessarily simply connected. This result turns out to be very useful, for example, in Hubbard-Schleicher-Sutherland's article [HSS]. There, the authors explain how to find all the roots of a polynomial, using Newton's method.

Remark. Shishikura [Sh2] proved that any rational map having only one weakly repelling fixed point has a connected Julia set and thus, every Fatou component is simply connected. There are examples showing that in Shishikura's result, one cannot replace weakly repelling by virtually repelling. For example, the quadratic rational map $z \mapsto z+1+1 /(9 z)$ has only one virtually repelling fixed point: $-1 / 9$. The other fixed point, $\infty$, has multiplicity 2 and is virtually attracting. However, the Julia set is totally disconnected.

The second example is the following: for any integer $m \geq 2$, if $\Re\left(a^{m-1}\right)<-1 / 2$ or $\Re\left(a^{m-1}\right)>m / 2$, then the polynomial $z-z^{m}+a z^{m+1}$ has a connected Julia set. Indeed, this polynomial has only two fixed point: 0 which has multiplicity $m$ and residue fixed point index $a^{m-1}$ and $1 / a$ which has multiplicity 1 and residue fixed point index $-a^{m-1}$. If the hypothesis are satisfied, only one of them is virtually repelling and the basin of infinity is simply connected. Thus, the Julia set is connected. This result can be proved differently, arguing that the number of critical points contained in the immediate basin of attraction of a virtually attracting or indifferent multiple fixed point is greater than the multiplicity of the fixed point (see for example $[\mathbf{S h} 3],[\mathbf{B}]$ or $[\mathbf{B E}]$ ). 
Let us now mention a possible application of Theorem 3. The result we give can probably be improved, for example when a rational map restricts to an orientation preserving homeomorphism of an annular compact set.

Proposition 2. Let $f: \mathbb{P}^{1} \rightarrow \mathbb{P}^{1}$ be a rational map that restricts to an orientation preserving homeomorphism of a Jordan curve $\gamma$. Let $V$ be a connected component of $\mathbb{P}^{1} \backslash \gamma$. Then one of the following three cases must occur.

1. The curve $\gamma$ contains a fixed point of $f$.

2. The restriction of $f$ to $V$ is conjugate to a rotation.

3. The component $V$ contains a virtually repelling fixed point of $f$.

Corollary 1. If a rational map $f: \mathbb{P}^{1} \rightarrow \mathbb{P}^{1}$ has a fixed Herman ring $A$, each connected component of $\mathbb{P}^{1} \backslash A$ contains a virtually repelling fixed point.

In [Sh2], Shishikura proves that more generally, if a rational map $f$ has a periodic Herman ring, then the cycle of Herman rings must separate two weakly repelling fixed points. His proof is based on quasi-conformal surgery. We do not know whether Corollary 1 can be proved using Shishikura's arguments.

Question. If a rational map has a periodic Herman ring, does the cycle of Herman rings separate two virtually repelling fixed points?

Question. Assume $f$ is a rational map and $\left(\gamma_{1}, \ldots, \gamma_{k}\right), k>2$, is a cycle of disjoint Jordan curves. Besides, assume $f^{\circ k}: \gamma_{1} \rightarrow \gamma_{1}$ is orientation preserving and has irrational rotation number. Does the cycle of Jordan curves separate two weakly (or virtually) repelling fixed points?

Acknowledgements. The author wishes to express his gratitude to François Bertheloot, Julien Duval and Christian Henriksen for fruitful discussions.

\section{References}

[A] L. V. Ahlfors, "Conformal invariants: topics in geometric function theory", McGraw-Hill Series in Higher Mathematics, McGraw-Hill Book Co., New York-Düsseldorf-Johannesburg, 1973.

[B] W. Bergweiler, On the number of critical points in parabolic basins, Ergodic Theory Dynam. Systems 22(3) (2002), 655-669.

[BE] X. Buff and A. L. Epstein, A parabolic Pommerenke-LevinYoccoz inequality, Fund. Math. 172(3) (2002), 249-289. 
[C] C. CAmacho, On the local structure of conformal mappings and holomorphic vector fields in $\mathbb{C}^{2}$, in: "Journées Singulières de Dijon" (Univ. Dijon, Dijon, 1978), Astérisque 59-60, Soc. Math. France, Paris, 1978, pp. 3, 83-94.

$[\mathrm{DH}]$ A. Doundy And J. H. Hubbard, On the dynamics of polynomial-like mappings, Ann. Sci. École Norm. Sup. (4) 18(2) (1985), 287-343.

[HSS] J. H. Hubbard, D. Schleicher and S. Sutherland, How to find all roots of complex polynomials by Newton's method, Invent. Math. 146(1) (2001), 1-33.

[J] H. Jellouli, Indice holomorphe et multiplicateur, in: "The Mandelbrot set, theme and variations", London Math. Soc. Lecture Note Ser. 274, Cambridge Univ. Press, Cambridge, 2000, pp. 253-264.

[M] J. MiLnor, "Dynamics in one complex variable. Introductory lectures", Friedr. Vieweg \& Sohn, Braunschweig, 1999.

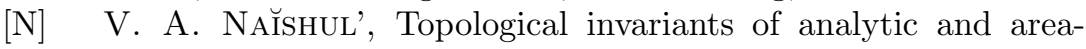
preserving mappings and their application to analytic differential equations in $\mathbb{C}^{2}$ and $\mathbb{C P}^{2}$, Trudy Moskov. Mat. Obshch. 44 (1982), 235-245 (in Russian).

[PM] R. PÉrez-Marco, Fixed points and circle maps, Acta Math. 179(2) (1997), 243-294.

[Pr] F. PRZYTYCKI, Remarks on the simple connectedness of basins of sinks for iterations of rational maps, in: "Dynamical systems and ergodic theory" (Warsaw, 1986), Banach Center Publ. 23, PWN, Warsaw, 1989, pp. 229-235.

[R1] P. Roesch, Topologie locale des méthodes de Newton cubiques, Thèse, École Normale Supérieure de Lyon (1997).

[R2] P. Roesch, Puzzles de Yoccoz pour les applications à allure rationnelle, Enseign. Math. (2) 45(1-2) (1999), 133-168.

[Sh1] M. SHISHIKURA, On the quasiconformal surgery of rational functions, Ann. Sci. École Norm. Sup. (4) 20(1) (1987), 1-29.

[Sh2] M. ShishikurA, Connectivity of the Julia set and fixed point, Preprint, Institut des Hautes Etudes Scientifiques, IHES/M/90/37 (1990).

[Sh3] M. ShishikurA, On the parabolic bifurcation of holomorphic maps, in: "Dynamical systems and related topics" (Nagoya, 1990), Adv. Ser. Dynam. Systems 9, World Sci. Publishing, River Edge, NJ, 1991, pp. 478-486. 
Université Paul Sabatier

Laboratoire Emile Picard

118, route de Narbonne

31062 Toulouse Cedex

France

E-mail address: buff@picard.ups-tlse.fr

Primera versió rebuda el 13 de març de 2002, darrera versió rebuda el 7 de maig de 2002. 